\title{
Simulation of Electro-thermal Ageing and Breakdown in Polymeric Insulation under High Frequency Trapezoidal- Wave Pulses
}

\author{
Zhou Zuo, Chenguo Yao \\ Chongqing University \\ State Key Laboratory of Power Transmission Equipment \& System Security and New Technology \\ Chongqing, 400044, China
}

\author{
L. A. Dissado, N. M. Chalashkanov and S. J. Dodd \\ University of Leicester \\ Department of Engineering \\ Leicester, LE1 7RH, UK
}

\begin{abstract}
A quantitative ageing model expressed in terms of two generic parameters, one describing the ability of local regions to resist degradation and the other describing their capacity to concentrate energy from the electric field, has been used to simulate the electro-thermal ageing of Polyethylene Terephthalate (PET) in pulsed trapezoidalwave electric fields. Allowance has been made for the dielectric heating produced by the alternate-polarity voltage pulses. It was found that for $90 \%$ of the lifetime the degradation was that of isolated regions. Only when the regions started to group to produce a local field in excess of a critical value did the degradation accelerate to failure in the form of a single track. The critical field is associated with the effective onset of irreversibility in the production of degradation, such that a local equilibrium state in the presence of the field was no longer possible regardless of the nature of the surrounding material. The temperature increases were found to be small and had very little influence upon degradation formation, though they had a minor influence on the initiation of runaway failure.
\end{abstract}

Index Terms - Simulation, ageing, electrical breakdown, polymers, polarization, dielectric heating, trapezoidal-wave pulses.

\section{INTRODUCTION}

IT is usual for manufacturers to be expected to guarantee their insulation systems for a defined lifetime in service conditions. For power transmission cables and H.V. capacitors, life predictions are typically made using one of a number of empirical life expressions, see [1]. More recently quantitative theoretical expressions have been derived for insulation lifetimes under $\mathrm{AC}$ and $\mathrm{DC}$ electrothermal ageing lifetimes with some success [2-4]. However the emergence of power electronic technologies [5-10] has posed several new problems because of their highly compact configurations $[5,11]$, dramatically larger thermal dissipation $[5$, 12-14], very rapid polarity changes (high $\mathrm{dv} / \mathrm{dt}$ ) and high repetition frequencies of applied field pulses [5-7] far in excess of the typical power system value of $50 / 60 \mathrm{~Hz}$. To limit high $\mathrm{dv} / \mathrm{dt}$ stress within the insulation, modular multilevel converter topologies with trapezoidal modulation have been proposed for medium and high voltage DC-DC transformers [15].

Here we develop a simulation model for electro-thermal aging under trapezoidal pulse voltage excitation which includes heating due to the dielectric losses produced by the consequent polarization currents. Use is made of a life expression based on the generic physical features determined from the work of [2-4]. The model is applied to electro-thermal aging of Polyethylene Terephthalate (PET), a material that can be used at temperatures well above $100{ }^{\circ} \mathrm{C}$ and has a good endurance in high fields, properties required for the insulation of power electronic devices. The aim of this work is not to replicate experimental data but to elucidate the way that ageing changes an insulation material during its working life under electrical pulse excitation and thermal stress, and determine the manner in which such changes brings the insulation to eventual failure. A quantitative physical model for ageing has been previously presented in [2] and there it was proven to fit the DC lifetime data for a set of $25 \mu \mathrm{m}$ thick PET films. By representing a $1 \mathrm{~mm}$ section of insulation as a mesh of 25 $\mu \mathrm{m}$ sections we are able to make use of this model to describe the evolution of change within each bond of the mesh in terms of the field and temperature that it experiences. The use of a trapezoidal voltage pulse excitation is typical of power electronics 
applications, but has the added advantage of introducing dielectric heating into the calculation hence allowing us to examine the influence of temperature increases due to polarization currents has upon the ageing process relative to that of electrical stress. The resulting simulations not only explain why it is notoriously difficult to detect irreversible material changes during ageing, but also why even a low density of changes can generate the local conditions necessary for the changes in one such region to result in failure by extending across the sample as a conducting path. By expressing the model utilized in terms of general energetic and morphological parameters that would be expected to apply to any insulating material, albeit with different values, the way is pointed towards an approach to the evaluation of material quality in terms of these parameters and their local distribution.

\section{BASIC AGEING MODEL}

Theories [2-4] of electro-thermal ageing in insulating polymers are based on the concept of field-driven chemical rate equations [16] to describe its progress. These are described in detail in [2-4] and so only a brief summary will be given here.

The state of the polymer is described by that of local elements whose morphology/chemistry can adopt one of two interconvertible configurations, the unaged (reactant) or deteriorated (product) state. The role of the electric field is twofold: (i) it reduces the energy barrier for the local reactions between the reactant and product state; (ii) it shifts the equilibrium towards a larger concentration of elements that have reacted through changes in the ground state energy of reactant and product state. The progress of the reaction is measured by the time-dependent concentration of elements that have reacted. If this reaches a critical level before equilibrium is attained, failure will occur.

The progress of the electro-thermal degradation reaction is defined in terms of a normalized variable $X$ [2], where $X=0$ corresponds to all reacting regions being in the reactant state and $X=1$ to all regions in the product state (deteriorated state).

The increase of $X$ over time, expresses the accumulation of degradation and failure is taken to occur when $X$ reaches or exceeds a critical value denoted by $A^{*}$. The time-evolution of $X$ is determined by:

$$
\frac{d X}{d t}=k_{f}-\left(k_{f}+k_{b}\right) X
$$

Here, $k_{f}$ is the forward rate constant and $k_{b}$ the backward rate constant. In the presence of an applied electric field $E$, they take the form [2]:

$$
k_{f}=\frac{k T}{h} \exp \left(-\frac{G^{\#}-C E^{b}}{k T}\right)
$$

and:

$$
k_{b}=\frac{k T}{h} \exp \left(-\frac{G^{\#}-\Delta}{k T}\right)
$$

Here $k$ and $h$ are the Boltzmann and Planck constants, $\Delta$ the energy difference between product and reactant ground states in the absence of the electric field $E, T$ the absolute temperature and $G^{\#}$ the activation free energy given by:

$$
G^{\#}=H^{\#}-T S^{\#}
$$

where $H^{\#}$ is the activation enthalpy and $S^{\#}$ is the activation entropy. In a constant DC field $E$, equation (1) gives an equilibrium conversion of $X=A_{e q}$, with:

$$
A_{e q}=\frac{k_{f}}{k_{f}+k_{b}}=\left[1+\exp \left(\frac{\Delta-C E^{b}}{k T}\right)\right]^{-1}
$$

Thus failure will only occur when $A^{*} \leq A_{e q}$. Although each theory gives different physical origins for, $H, C, \Delta, b$, and $A^{*}$, it is preferable to adopt the strategy used in $[17,18]$ of treating them as generic parameters, since their values cannot be determined by experiments other than endurance lifetimes and all theories can fit the field dependence of the characteristic endurance lifetime. Thus $C E^{b}$ can be regarded as a measure of the field energy concentration at a specific location that is available to drive the reaction since the larger $C E^{b}$ the smaller the energy barrier to modification and the greater the amount of change at equilibrium, and $A^{*}$ is a measure of the ability of given location to resist deterioration, i.e. the bigger $A^{*}$ the greater the amount of change required for irreversible deterioration $[19,20]$.

\section{HEAT DISSIPATION IN TRAPEZOIDAL WAVE FIELD PULSES}

A typical schematic for trapezoidal wave pulse fields is shown in figure 1. Joule heating due to DC currents will occur to its largest extent on the constant stress sections of the pulse. However the DC conductivity of insulating dielectrics is very small, typically less than $10^{-14} \mathrm{~S} / \mathrm{m}$ [21] so the heat density dissipated per pulse is exceedingly small. For example, $\sigma \approx$ $6.5 \times 10^{-17} \mathrm{~S} / \mathrm{m}$ for PET at $110^{\circ} \mathrm{C}$ (estimated from [22]), which is the material used here for the simulation, gives a heat density of $\sigma E^{2} t_{p} \sim 5 \times 10^{-6} \mathrm{~J} / \mathrm{m}^{3}$ per pulse when the field is 40 $\mathrm{MV} / \mathrm{m}$ and $t_{p}=5 \times 10^{-5} \mathrm{~s}$. Almost all of the heating therefore comes from the polarization currents produced by the polarity reversal inherent in field pulse excitation.

The dielectric heating due to the polarization currents is obtained by the following method. First linear response theory [23] is used to obtain the time-dependent change in polarization, $P(\mathrm{t})$ :

$$
P(t)=\varepsilon_{0} \int_{-\infty}^{t} \phi\left(t-t^{\prime}\right) E\left(t^{\prime}\right) \mathrm{d} t^{\prime}
$$

Here $\varepsilon_{0}$ is the permittivity of free space, and $\phi(t)$ the response function. The exact form of $\phi(t)$ varies with the specific dielectric relaxation [24], but a simple exponential form contains the basic features, i.e. an amplitude (contribution to the static relative susceptibility $\chi_{0}$ ) and a relaxation time ( $\tau=1 / \omega_{p}=1 /\left(2 \pi f_{p}\right) ; f_{p}=$ loss peak frequency) and is sufficient to give a good estimation of dielectric heating in the simulation.

$$
\phi(t)=\frac{\chi_{0}}{\tau} \exp \left(-\frac{t}{\tau}\right)
$$

Since the pulse is of finite duration, the polarization will not normally reach equilibrium after the time $t_{r}+t_{p}$ (see Figure 1). Its value, $P_{0}$, can be obtained by equating it with $P_{0}+\Delta P\left(t_{r}+t_{p}\right)$, where $\Delta P\left(t_{r}+t_{p}\right)$ is the change in polarization 
during the switch in polarity calculated from equation (6) for the time-dependent field of the pulse.

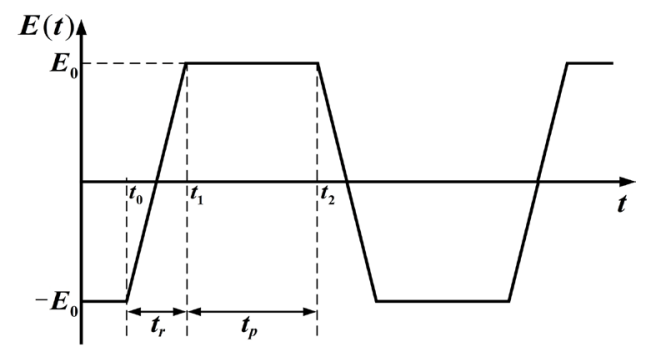

Figure 1. Schematic of trapezoidal-wave pulse field.

The next stage is to calculate the time-dependent polarization current density, $J(t)$, by differentiating $P(t)$ with respect to time, i.e. $J(t)=\mathrm{d} P(t) / \mathrm{d} t$. The total energy density dissipated during a pulse (i.e. at $t=t_{2}$ ) is the sum of, $Q_{r}$, from the rise period where the current density is denoted by $J_{r}(t)$ :

$$
Q_{r}=\int_{0}^{t_{r}} J_{r}(t) \Delta E_{r}(t) \mathrm{d} t
$$

and, $Q_{p}$, from the period of constant field:

$$
Q_{p}=\int_{t_{r}}^{t_{r}+t_{p}} J_{p}(t) E_{0}(t) \mathrm{d} t
$$

The polarization, polarization currents, and heat dissipated are sensitive to the values of the rise time and pulse duration with respect to the relaxation time, $\tau$, of the dielectric response, which is usually strongly dependent upon temperature. In order to illustrate this sensitivity, we have made a set of calculations for a number of different rise times $\left(t_{r}\right)$ taking PET to be the insulating dielectric. PET has two dielectric relaxations [25-27] the $\alpha$-relaxation process which is the glass-rubber transition in the amorphous fraction, and the $\beta$-relaxation which remains active in the glass region, see Table 1. Since the $\beta$-relaxation occurs in the $10-50 \mathrm{MHz}$ range for temperatures between 75 and $110{ }^{\circ} \mathrm{C}$, its contribution to the dielectric heating will effectively be completed by the end of a pulse of $50 \mu$ s duration at these temperatures.

Table 1. Parameter values for dielectric response of PET [27].

\begin{tabular}{ccccc}
\hline$T\left({ }^{\circ} \mathrm{C}\right)$ & $f_{p}(\mathrm{~Hz})^{1}$ & $\chi_{0}(\alpha)^{1}$ & $f_{p}(\mathrm{~Hz})^{2}$ & $\chi_{0}(\beta)^{2}$ \\
\hline 85 & 76 & 2.08 & $1.83 \times 10^{7}$ & 1.17 \\
95 & 3372 & 1.84 & $3.08 \times 10^{7}$ & 1.29 \\
105 & 57600 & 1.61 & $5.05 \times 10^{7}$ & 1.39 \\
\hline
\end{tabular}

${ }^{1}$ Parameter Values for the $\alpha$-relaxation process.

${ }^{2}$ Parameter Values for the $\beta$-relaxation process.

On the other hand, the contribution of the $\alpha$-relaxation will be sensitive to $t_{r}$ and $t_{p}$ and so the calculations in this section will be restricted to the effect of this response. The results given in figure 2 show that the variation in rise time between $0.1 \mu$ s and $2 \mu \mathrm{s}$ at any given temperature in the range $85^{\circ} \mathrm{C}$ to $105^{\circ} \mathrm{C}$ has very little effect, but the reduction in the relaxation time, $\tau$, as the temperature increases is very significant. For example, at $85{ }^{\circ} \mathrm{C}$ where $f_{p}$ is small and $\tau>t_{r}+t_{p}$ the polarization does not reach equilibrium during the duration of the pulse and the polarization current density is small and nearly constant throughout the pulse, as shown in figure 2a. When $T=105{ }^{\circ} \mathrm{C}$, figure $2 \mathrm{c}$ nearly all the polarization takes place during the rise time and it comes almost into equilibrium by the end of the pulse. Now the polarization current density is sharply peaked near the end of the rise time $t_{r}$ with a maximum value about 500 times its value at $T=85{ }^{\circ} \mathrm{C}$. Despite the short duration of the rise time $\left(t_{r}<<t_{p}\right)$, the increase in maximum polarization current as the temperature increases dominates over the reduction in effective time of application and the value of the heat dissipated per pulse by the $\alpha$-relaxation $\left(Q_{\alpha}\right)$ becomes larger as the temperature increases. For example, with $t_{r}=2 \mu$ s the values of $Q_{\alpha}$ are $Q_{\alpha}=1422 \mathrm{~J} / \mathrm{m}^{3}$ at $T=85{ }^{\circ} \mathrm{C}$; rising continuously to $Q_{\alpha}=49350 \mathrm{~J} / \mathrm{m}^{3}$ at $T=105{ }^{\circ} \mathrm{C}$. Over this temperature range, the contribution of the $\beta$-relaxation $\left(Q_{\beta}\right)$ will rise from $33410 \mathrm{~J} / \mathrm{m}^{3}$ to $39470 \mathrm{~J} / \mathrm{m}^{3}$. There will therefore be a positive feedback in the dielectric heating, with its consequent temperature increase increasing further the heat dissipated. However the temperature rise can be expected to be limited by thermal conduction.

\section{NUMERIC SIMULATION OF AGING AND BREAKDOWN}

PET is the material chosen for simulation because it can be used at high temperatures up to $180^{\circ} \mathrm{C}$ as required for pulsed power systems. Local variation of material properties means that the aging parameters of the aging model have to be described by distributions and their characteristic values. These are known for the DC aging of $25 \mu \mathrm{m}$ thick films of PET [2, 28, 29] and for this reason we adopt a simulation grid whose mesh size is $25 \mu \mathrm{m}$. A $50 \times 40$ two-dimensional (2D) grid is used corresponding to a $1.25 \mathrm{~mm} \times 1 \mathrm{~mm}$ section of a sample of PET. The characteristic parameter values are given in Table 2. We have taken $\Delta=0$ so as to simplify the calculation because its value is much less than that of $H^{\#}$ and it only has an influence at fields close to the threshold value below which field-dependent changes reach equilibrium and do not cause irreversible damage [19, 20]. Previous work [30] has shown that only distributions of $C$ and $A^{*}$ are significant for the lifetime distribution and hence we have assumed that only these parameters will vary on the $25 \mu \mathrm{m}$ scale of the grid. This variation is represented by the assignment of $C$ and $A^{*}$ values to each grid-bond randomly selected from a distribution shape that approximates the experimentally determined distributions, as in [17, 18], and that possesses the experimental characteristic values. These are a uniform distribution of $C / k$ truncated at 1.0 and $\sim 2.2 \mathrm{~K}(\mathrm{~m} / \mathrm{MV})^{b}$, and $A^{*}$ from a uniform distribution between the limits of 0.01 and 0.96. This approach simplifies the simulation while still allowing the trends in behaviour to be established.

Table 2. Characteristic values of the parameters for the DC life of PET [2]

\begin{tabular}{lll}
\hline Parameters & Units & Characteristic value \\
\hline$H^{\# / k}$ & $\mathrm{~K}^{1}$ & 17762 \\
$S^{\#}$ & $\mathrm{~J} / \mathrm{K}$ & $-9.1 \times 10^{-25}$ \\
$b$ & - & 1.556 \\
$C / k$ & $\mathrm{~K}(\mathrm{~m} / \mathrm{MV})^{b}$ & 1.582 \\
$A^{*}$ & - & 0.485 \\
\hline
\end{tabular}

${ }^{1}$ The unit denoted $\mathrm{K}$ renresents the temnerature in Kelvin. 


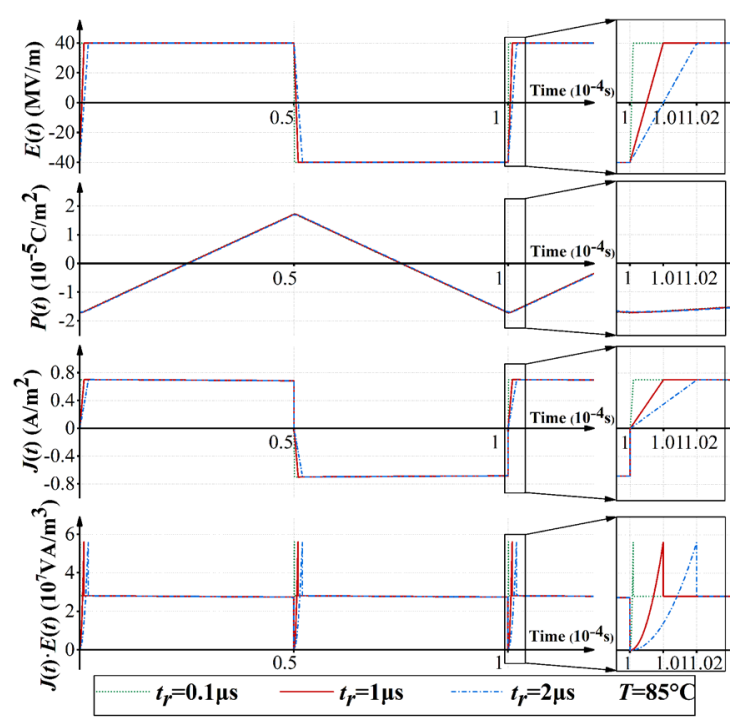

(a)

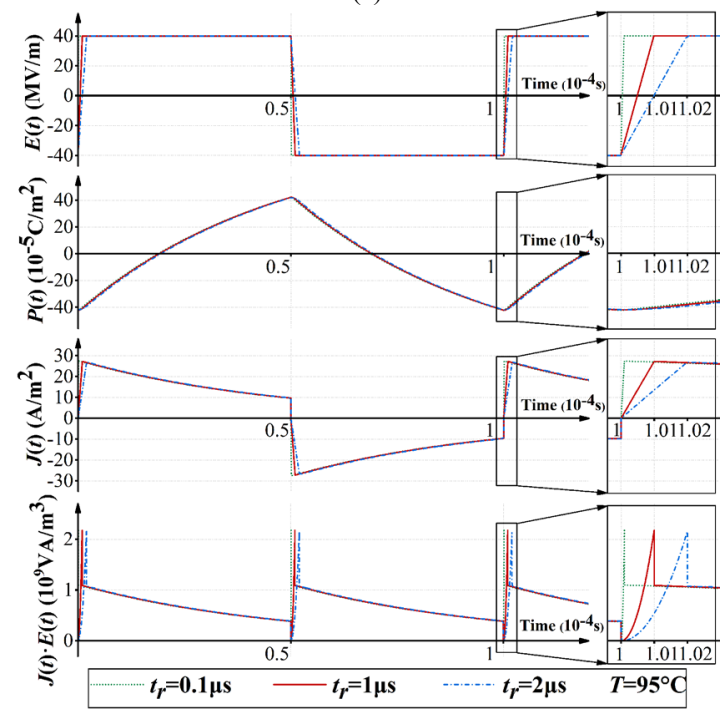

(b)

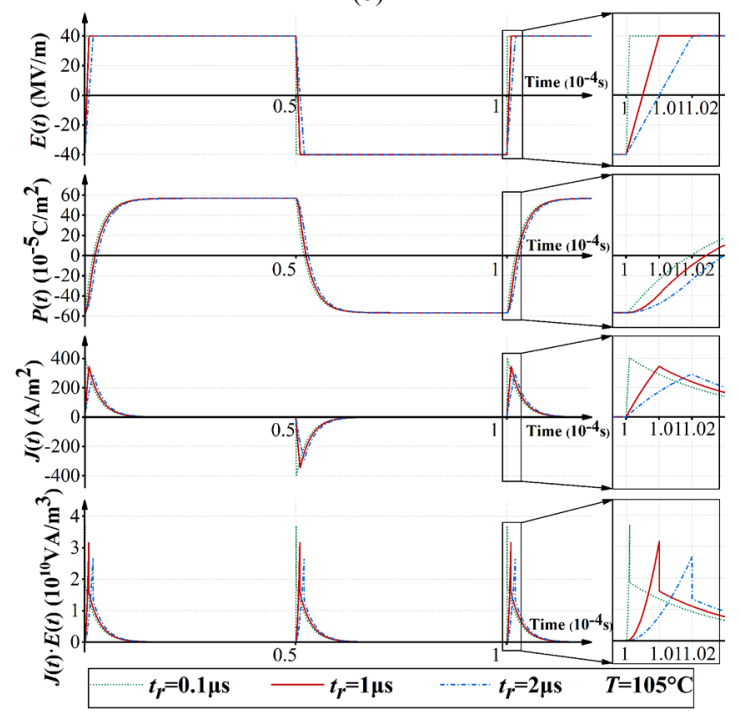

(c)

Figure 2. Calculations of electric field $E(t)$, polarization $P(t)$, current density $J(t)$, and heating rate $J(t) \cdot E(t)$, for the $\alpha$-relaxation process of PET under trapezoidal wave pulse excitation $\left(E_{0}=40 \mathrm{MV} / \mathrm{m} ; f=10 \mathrm{kHz}\right)$ with rise times $0.1 \mu \mathrm{s}, 1 \mu \mathrm{s}$ and $2 \mu \mathrm{s}$. (a) $\mathrm{T}=85^{\circ} \mathrm{C}$, (b) $\mathrm{T}=95^{\circ} \mathrm{C}$, (c) $\mathrm{T}=105^{\circ} \mathrm{C}$.
In the simulation, the 2D-grid is placed between two equipotential lines representing plane electrodes. Both electrodes are taken to be heat sinks at a fixed ambient temperature. The sequence of trapezoidal wave field pulses is applied to the top electrode and the lower electrode is taken to be at ground potential. Since the simulation grid is much smaller than a real sample, edge effects must be removed to prevent the simulation from generating unreal results. We therefore use periodic boundary conditions, whereby the temperatures and fields generated along one edge as a result of the progress of heating and deterioration are made the same as those along the other edge. In addition, the domain where the ageing is simulated is restricted to the inner $40 \times 40$ grid, so that no aging occurs in the outer domains alongside the edges with $5 \times 41$ grid bonds and thus they do not contribute artefacts to the progress of deterioration as a result of the imposed boundary conditions.

Heat is transferred to the grid-bonds via the polarization currents in them, which gives the heat dissipated per pulse $Q$ $\left(Q=Q_{r}+Q_{p}\right)$. The temperature increase depends upon, the specific heat $C_{p}$, the density $\rho$, and the coefficient of thermal conduction $k$, through:

$$
\rho C_{p} \frac{\partial T}{\partial t}-\nabla \cdot(k \nabla T)=Q
$$

Values for $C_{p}, \rho$, and $k$ for PET are taken from [31].

At the beginning of the simulation, values of $C$ and $A^{*}$ are assigned to each grid-bond of the matrix, which are taken to be unaged non-conducting capacitors with $X=0$. The ageing in each grid-bond depends upon the assigned local values of $C$ and $A^{*}$, the local field, and the local temperature determined from the polarization current heating and the heat conduction equation. Because $t_{r}$ is small compared to $t_{p}$, the whole of the ageing is assumed to take place in the constant field during the dwell time $\left(t_{p}\right)$ of the pulse. Use of equations (1) and (5) allow the progress of the ageing in any particular matrix bond to be written as a recursion equation for the value of $X$ after $n$ pulses, $X^{n}$, in terms of the progress at the end of the previous pulse $X^{n-1}$ given by:

$$
X^{n}=A_{e q}\left\{1-\exp \left[-\left(k_{f}+k_{b}\right) t_{p}\right]\right\}+X^{n-1} \exp \left[-\left(k_{f}+k_{b}\right) t_{p}\right]
$$

The progress of the ageing is written in this way to allow account to be taken of changes, such as field distribution due to a bond failure elsewhere on the grid, and increases in temperature due to the polarization currents.

When the ageing progress, $X$, in a bond (or bonds) reaches the value of $A^{*}$ assigned to the bond, i.e. $X \geq A^{*}$, this bond (or bonds) is (are) regarded to have failed and become a conductor as would be the case for the $25 \mu \mathrm{m}$ films of [2]. As in $[17,18]$, the field in the failed matrix bond is assumed to be zero and consequently the field distribution is modified and must be recalculated using the Laplace equation:

$$
\nabla^{2} V=0
$$

where $V$ is the potential. Thus the initial uniform field distribution is distorted when the first bond fails and the field becomes enhanced around the failed bonds. As a result, the rate of aging in the un-failed grid-bonds is modified. Since $Q$ 
is a function of the field and the temperature in the un-failed grid-bonds the heating is also driven by bond failure elsewhere that enhance the local field, and it thus provides a feedback to the progress of ageing resulting in a dynamic electro-thermal process for the overall ageing of the whole matrix.

\section{RESULTS}

Simulations have been performed for PET at an ambient temperature of $110{ }^{\circ} \mathrm{C}$ with an applied trapezoidal pulse field of magnitude $40 \mathrm{MV} / \mathrm{m}$; frequency $10 \mathrm{kHz}$; rise time of $1 \mu \mathrm{s}$. Both $\alpha$ and $\beta$ relaxations are included in the calculation of heating. The data of [25-27] has been used to obtain Arrhenius plots of the relaxation times and amplitudes, which are extrapolated as necessary to obtain their values at the local temperature of the bond for which the heating is being calculated. Calculation of the temperature for a uniform field of $\pm 40 \mathrm{MV} / \mathrm{m}$ and ambient temperature of $110{ }^{\circ} \mathrm{C}$ shows an increase along the central row of only $\sim 0.055{ }^{\circ} \mathrm{C}$, with the increase dropping continuously to zero on moving away from this row to the electrodes. This implies that in the absence of field enhancement by material imperfections dielectric heating will have very little effect on the formation of the first failed matrix bond.

Simulations have been carried out for three different ways of representing variations in material ageing parameters at the $25 \mu \mathrm{m}$ scale. As in $[17,18]$, either $A^{*}$ is varied with $C$ held at its characteristic value or $C$ is varied with $A^{*}$ held at its characteristic value. The third scheme allows variation of both $A^{*}$ and $C$ for the whole matrix. The ageing is followed through the progressive increase in the number of failed bonds up to failure when a conducting path is formed across the matrix, Figure $3 \mathrm{a}$. The number of bonds given in the figure is the average over ten repeat simulations for each condition, each time with a new assignment of parameter values to the matrix bonds. As in [17, 18], the number of failed bonds in each case increased very slowly for about $90 \%$ of the lifetime and then increased rapidly as the growth of a nearly un-branched failure structure accelerated across the matrix. Figure $3 \mathrm{a}$ also reveals that the ageing lifetime is shortened when $A^{*}$ is varied in the matrix bonds as compared to when only $C$ is varied, and that the lifetime is substantially the same whether $C$ is varied together with $A^{*}$ or set at its characteristic value. In figures $3 \mathrm{~b}$ $3 \mathrm{~d}$, we show the time evolution of the maximum local electric field within the simulation matrix as the ageing deterioration progresses, with figure $3 \mathrm{~b}$ referring to a matrix where only $A^{*}$ is spatially varied, Figure $3 \mathrm{c}$ to a matrix in which only $C$ is varied, and figure $3 \mathrm{~d}$ a matrix in which both $A^{*}$ and $C$ are spatially varied. The average maximum local field at three distinct phases of deterioration is noted in each figure. The field $\bar{E}_{1}$ is that at which an isolated deteriorated bond grows by the failure of one or two neighbors. Not all isolated bonds achieve this status, but several are formed. However, the extension of the group to several adjoining bonds is a rare event with one or two such groups being generated. When the maximum field at the tip of one of these groups reaches $\bar{E}_{2}$ the extension starts to run away to the failure of the matrix as a path of failed bonds is formed across the matrix. At a field of $\bar{E}_{3}$ only one extending group is present and the extension is too rapid to resolve into the addition of single extra failures and the failure path accelerates across the matrix as would be expected for a deterministic breakdown mechanism, with breakdown taking only about $1 \mathrm{~s}$ following the achievement of this local field. It can be seen that regardless of the composition of the matrix (i.e. its spatial distribution of parameters) and the consequent lifetime, the average values of the three fields have almost the same value, suggesting that these values can be regarded as critical values that are necessary for the onset of each succeeding stage.

Repeat simulations have been carried out, each time with a new assignment of parameter values to the matrix bonds. The lifetimes, $L$, obtained are fitted to a Weibull distribution [21] whose cumulative probability function is given by:

$$
P_{F}=1-\exp \left[-\left(L / L_{c}\right)^{\beta}\right]
$$

where $L_{c}$ is the characteristic life, and $\beta$ is the parameter that determines the shape of the probability density function. The probability plot of Figure 4 confirms that the lifetime distribution for spatial inhomogeneity in $A^{*}$ is shifted to lower values compared to the one for spatial inhomogeneity in $C$ only. Thus, bonds (regions) with a weak resistance to ageing deterioration determine the lifetime, rather than the bonds with a strong ability to concentrate energy from the electric field. However, it should be noted that, as in $[17,18]$, most of the failed bonds remain isolated for the greatest part of the lifetime rather than immediately initiating a track that develops into matrix failure, see figure $5 \mathrm{a}$. This is the case even when these bonds fail early due to a small resistance to deterioration $\left(A^{*}<<0.485\right)$ as when there is inhomogeneity in $A^{*}$. It is clear that ageing failure is not initiated by the very weakest region. In the following we shall argue that instead the local failure of weak regions combine to enhance the local field at one of the regions sufficient to cause it to extend and eventually runaway to matrix failure.

Figures 5 and 6 show the evolution of the degradation and the corresponding field and temperature distributions during the extension and final runaway stage of ageing, for a simulation with spatial variation of both $A^{*}$ and $C$. The particular example chosen shows a failure structure initiating from degradation at the upper electrode, but this location is not common with most failures initiating in the body of the matrix.

During the extension period, prior to runaway more than one group of bonds extend (figure 5a: left). However the group that will generate the runaway has a field at the tip of its structure that increases to the greatest extent $(\sim 3 \times)$ and influences more neighboring bonds. As the extension of this group starts to runaway (figure 5a: right), the field rises enormously $(\sim 6 \times)$, see figures $3 b-3 d$. This increase of local field is clearly the major factor in the development of the ageing from isolated degradation to final failure.

During this process, the temperature distribution is also altered. As the degraded group starts to extend, the maximum temperature becomes located in a region of the center ahead of 


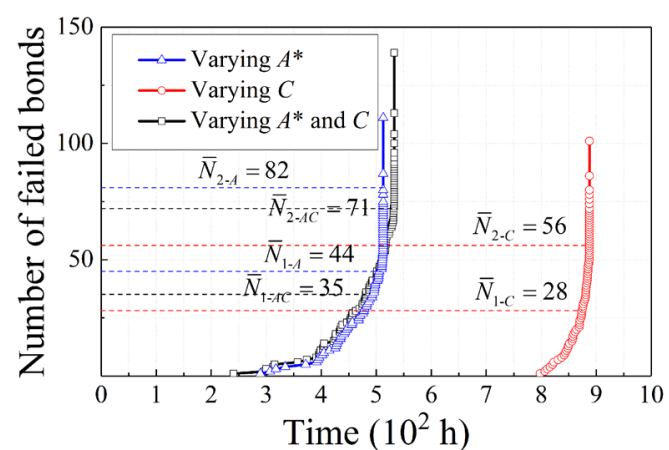

(a)

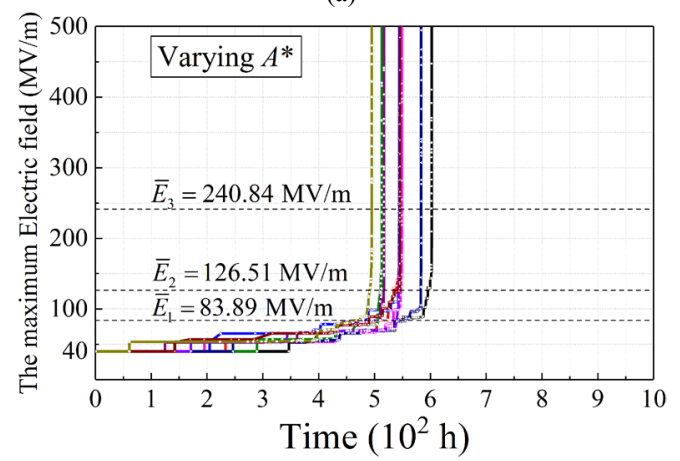

(b)

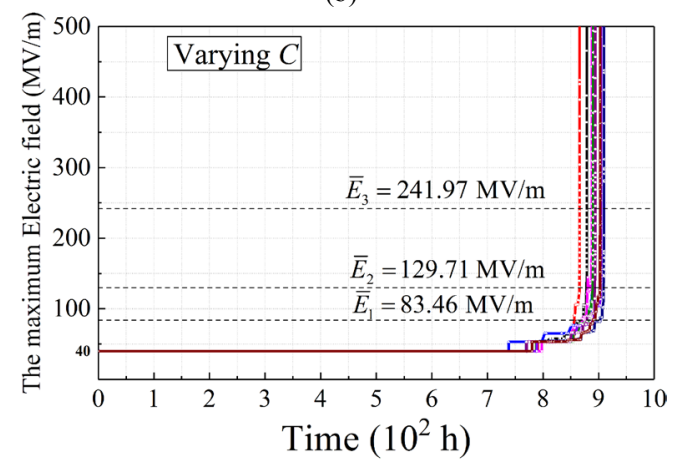

(c)

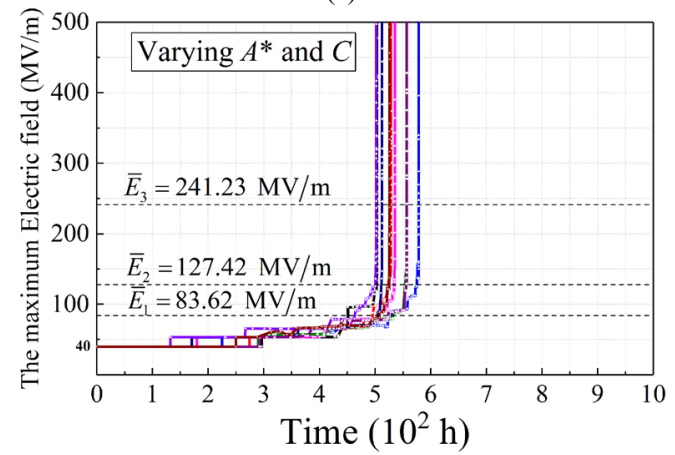

(d)

Figure 3. (a) The evolutions of failed bonds in the three schemes. $\bar{N}_{1-A}$, $\bar{N}_{1-C}$ and $\bar{N}_{1-A C}$ are average values of the number of failed bonds in the time up to runaway obtained from repeat simulations. $\bar{N}_{2-A}, \bar{N}_{2-C}$ and $\bar{N}_{2-A C}$ are average values at the start of runaway. (b), (c), and (d) show the evolution of maximum local field. $\bar{E}_{1}, \bar{E}_{2}$ and $\bar{E}_{3}$ are the average values of the onset field, $E_{1}$ : the extension field, $E_{2}$; and final runaway field, $E_{3}$.

the extending group (figure $5 \mathrm{~d}$ : middle), as a consequence of the divergent field around the tip of the extending group of bond failures. Although the temperature increases above ambient is only $0.1{ }^{\circ} \mathrm{C}$, this may have the effect of increasing the degradation rates in the bonds beyond the extending group of failures and hence contribute to their further extension. When runaway is initiated the maximum temperature and electric field are located in the same region (Figures $5 \mathrm{c}-5 \mathrm{~d}$ : right), and they may, therefore, combine to begin the runaway behaviour. However, the time between runaway initiation and failure is shorter than $1 \mathrm{~s}$, which is much shorter than the heating rate and thermal conduction so that when a failure occurs the region of maximum temperature is still located in the center near to the breakdown path. The final stage of failure is therefore not associated with a thermal runaway process (see [21]). Furthermore, the temperature increase throughout the ageing is small and thus the increase in the local electric field is the major factor in initiating runaway as long as there is no thermal inhomogeneity present.

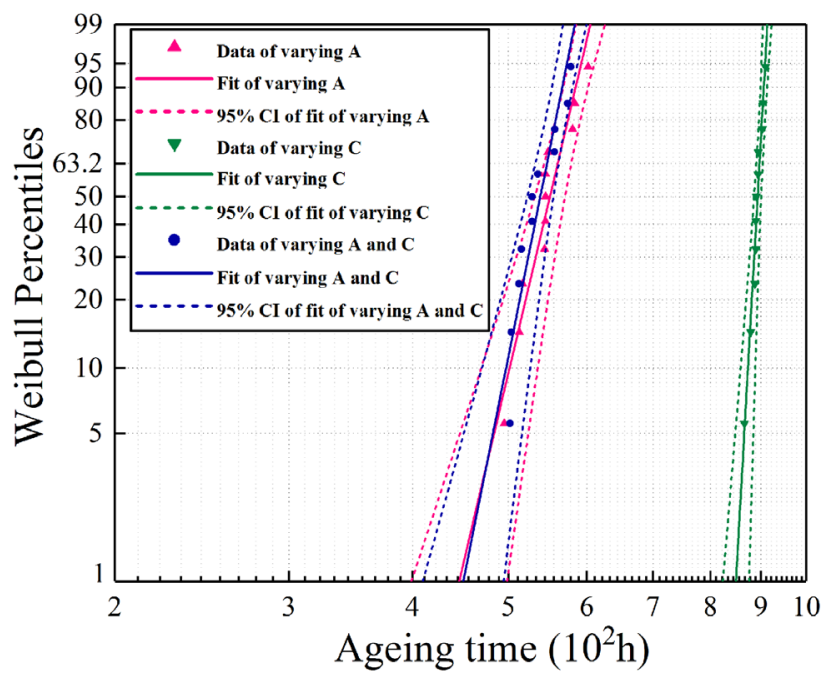

Figure 4. Weibull plots [1] of lifetimes with $95 \%$ confidence intervals using the least squares estimation method. The characteristic lives of the three simulation schemes are: $L_{c-A}=2022000 \mathrm{~s}(\Delta), L_{c-C}=3229000 \mathrm{~s}(\boldsymbol{\nabla})$, and $L_{c-A C}=1974000 \mathrm{~s}(\bullet)$. The values of the Weibull shape parameters, $\beta$, (gradient of the fitted line) are: $\beta_{A}=19.05, \beta_{C}=90.36$, and $\beta_{A C}=21.72$.

\section{DISCUSSION}

Figure 3 shows that the onset field, $E_{l}$, for the formation of degradation groups, and that for the extension of the groups, $E_{2}$, are independent of the composition of the simulation matrix. These are therefore critical fields for the onset of runaway failure such as in deterministic breakdown [21].

This shows for the first time that electro-thermal ageing is a process whereby progressive local degradation continually builds up the maximum local field to a critical level at which a deterministic runaway failure is switched on. Macroscopic defects, in contrast, give immediate field enhancement to the critical value [30], and the time to failure is governed by the generation of a conducting path. A value of $83.5 \mathrm{MV} / \mathrm{m}$ for $E_{1}$ together with $C$ set at its characteristic value gives $A_{e q}=0.98$, i.e. it is not possible for a bond to come into equilibrium with the field without being degraded. Thus the ability of neighboring bonds to resist degradation is ineffective at this field if their ability to concentrate field energy is at or greater than the characteristic value. At $E_{1}$, the rate constants $k_{f}+k_{b}$ 

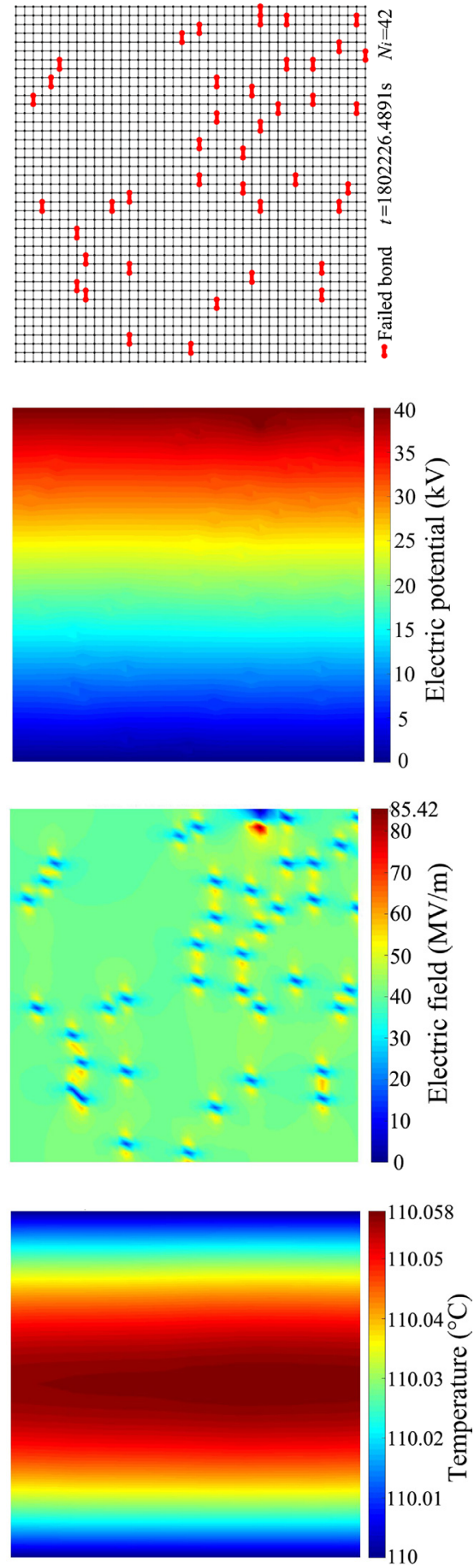

Figure 5. The evolution of: (a) failure structure; (b) electric potential; (c) electric field and (d) temperature - (left) at the beginning of the formation of degradation groups; (middle) short time after that; (right) before the final failure.

that determine the progress of the reaction equation (11) are about ten times their value when the simulation starts in the uniform field $E=40 \mathrm{MV} / \mathrm{m}$, and $k_{f} \sim 10-50 k_{b}$. At $E_{2}=128$ $\mathrm{MV} / \mathrm{m}, A_{e q}=0.99$ even when the neighboring bonds have the smallest values of $C$ allowed in the simulation. Now there is no longer any neighboring bond that can reach equilibrium without being degraded whatever their value for $C$. In this case, $k_{f}+k_{b}$ has increased further to approximately 600 times their initial value and $k_{f} \sim 150-2500 k_{b}$. Therefore, once the

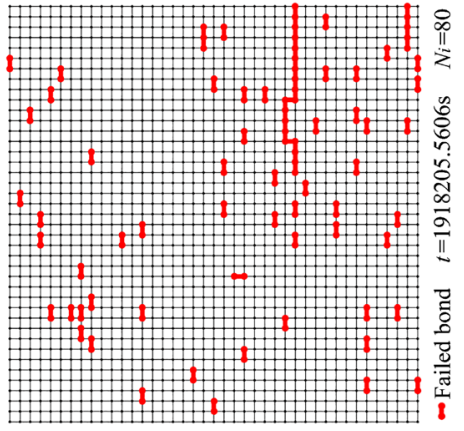

(a)

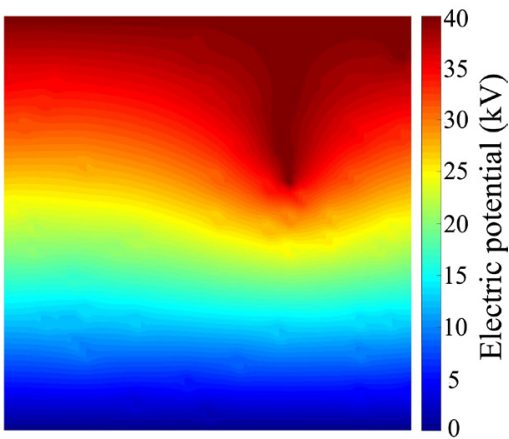

(b)

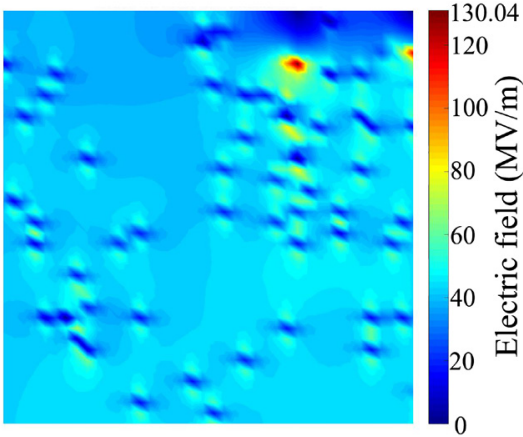

(c)
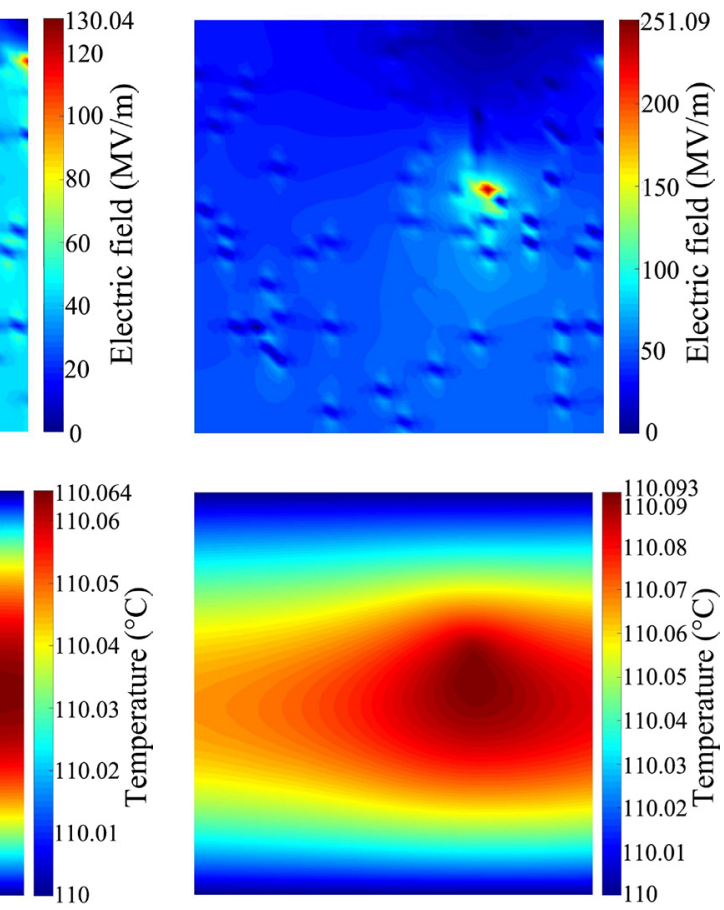

(d) local field is large enough that the forward reaction rate becomes substantially greater than the backward rate, grouping of the local degradation starts to occur. When this field becomes large enough that the forward rate massively dominates the backward rate runaway will occur, i.e. the onset of runaway failure is when the field becomes large enough that the reaction becomes effectively irreversible. These values of critical field will remain almost the same whether or not $A^{*}$, or $C$, or both, are varied on the simulation matrix. Thus at fields where mechanisms that 
lead to deterministic breakdown are stable i.e. their positive and negative feedback processes come into equilibrium [21], the forwards and backwards reactions of electrothermal ageing produce isolated local degradation, which builds up the maximum local field until it is large enough initiate an irreversible local runaway process.

The runaway process only takes about $1 \mathrm{~s}$ and together with the extension of degradation groups occupies only the final $9-10 \%$ of the total lifetime. The ageing lifetime is thus determined by the time taken for the field to be enhanced to the critical value, during which isolated degradation regions are formed, as found in [17, 18]. It will therefore be difficult to detect and monitor changes due to ageing. The rate of increase of maximum field is also weak in this stage of ageing, see figure $3 \mathrm{~b}$ to $3 \mathrm{~d}$, and so macroscopic changes such as in the conductivity will also be difficult to observe. So monitoring the progress of ageing through following changes in physical properties will not be easy, though there have been some attempts $[32,33]$.

Material quality is clearly an important issue. Figures $3 \mathrm{~b}$ to $3 \mathrm{~d}$ and Figure 4 show that spatial variation in the resistance to degradation $\left(A^{*}\right)$ is the most influential factor in reducing the ageing lifetime. The isolated degradation corresponds to small local values of $A^{*}$, i.e. locally weak regions in an electrical sense. When these regions are randomly distributed in space and do not possess a connected structure, they do not extend further until the local field reaches the critical value. When $A^{*}$ is spatially uniform and the only spatial inhomogeneity is in variation of the local ability to concentrate the field energy via $C$, the lifetime approximately doubles. This result shows that spatial uniformity in the morphological factors that may influence $A^{*}$, such as local density and mechanical strains is necessary for a good insulating dielectric, as well as the need for a good average value of these properties.

The simulations also show that in the absence of thermal inhomogeneity dielectric heating gives only a small increase in temperature that is not related to the formation of the isolated degradation that occurs during the major part of the lifetime. Only when degradation groups begin to form does the temperature increase have an effect. At this time, slightly higher temperatures are produced just in advance of the extending group, which serves to aid the extension. At the time when a runaway is initiated, the higher temperatures and fields have the same location, and hence there will be some reinforcement of the extension of the failure track. However, the runaway failure is faster than the heating rate and thermal conduction, and the heating plays no further role in the final failure, which is an electrical rather than thermal runaway. The outcome may be different if there is inhomogeneity in thermal factors such as the specific heat and permittivity, which requires further simulation.

The lifetime distributions of Figure 4 have a large Weibull shape parameter corresponding to distributions that are sharply peaked and hence almost deterministic, as also implied in the simulations of [18]. Experimental data gives much wider distributions with values of the shape parameter in the range $1<\beta<3$ (see [30]). The most probable reason for this discrepancy is the approximate form of the parameter distributions and limited range of parameter values for $C$. This means that extremely large values of $C$ or very small values of $A^{*}$ are not present in the matrix and thus the rapid degradation that they allow does not occur. Further simulations with selections from more realistic distributions will be needed to confirm this speculation, especially as many isolated degradation regions are formed before the onset of failure and thus the lifetime is not dependent on a single bond with the most extreme value of $A^{*}$ or $C$. It is also possible that spatial inhomogeneity due to structures on a grosser scale than the $25 \mu \mathrm{m}$ used here may play a role. Further simulations are required to clear up this point.

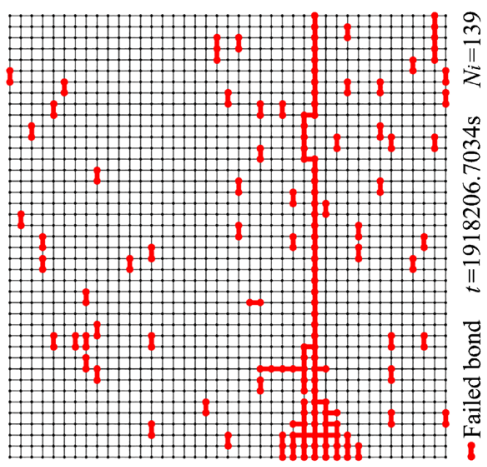

(a)

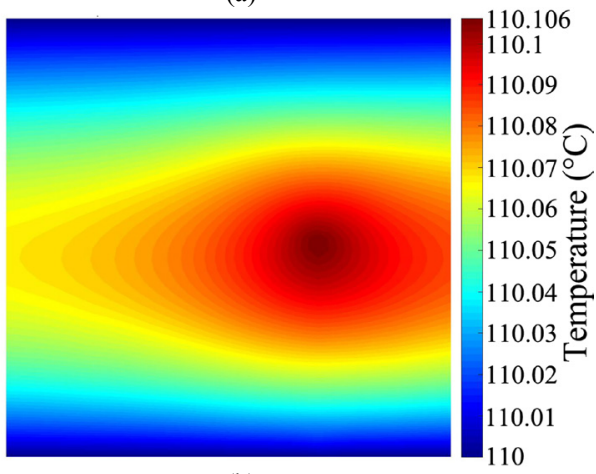

(b)

Figure 6. The final breakdown: (a) the failure structure, and (b) the temperature field.

\section{CONCLUSIONS}

Simulation of electro-thermal ageing under alternate polarity trapezoidal-wave pulse excitation $( \pm 40 \mathrm{MV} / \mathrm{m})$ in a $1.25 \mathrm{~mm}$ by $1 \mathrm{~mm} 2 \mathrm{D}$-section of PET at $T=110{ }^{\circ} \mathrm{C}$ showed that:

1) The ageing mechanism is one in which isolated local degradation progressively increases the maximum local field up to a critical value at which degradation groups are formed that lead to a runaway failure.

2) The critical field is associated with the effective onset of irreversibility in the local degradation rate process, such that no region along the runaway path can come into equilibrium with the applied field without being degraded.

3) The ageing lifetime is governed by the formation of isolated local degradation, which occupies $90 \%$ of the total 
time.

4) The isolated degradation is associated with locally 'weak' regions (small $A^{*}$ ) or regions of high concentration of field energy (large $C$ ).

5) Spatial inhomogeneity in the ability of local regions to resist degradation is the most important factor in lowering the lifetime. Only when the associated parameter $\left(A^{*}\right)$ is spatially uniform does the spatial variation in local concentration of field energy become important.

6) In the absence of spatial inhomogeneity in specific heat and thermal conductivity, the dielectric heating caused by trapezoidal-wave pulse excitation only increases the temperature by a small amount $\left(0.05-0.1{ }^{\circ} \mathrm{C}\right)$ and has no discernible effect for the $90 \%$ of the lifetime during which the degradation is locally isolated.

7) Dielectric heating has a small effect in aiding the onset of the runaway failure, but does not perform as a thermal runaway.

These considerations also allow us to conclude that a good insulating dielectric must not only possess good macroscopic average values of properties related to its electro-thermal endurance, but that these properties must have as little spatial inhomogeneity as possible.

\section{ACKNOWLEDGMENT}

This work was supported by the National Natural Science Foundation of China under Grant No. 51321063.

\section{REFERENCES}

[1] L. Simoni, "A General Approach to the Endurance of Electrical Insulation under Temperature and Voltage" IEEE Trans. Electr. Insul., Vol.16, pp277-289, 1981

[2] L. A. Dissado, G. Mazzanti and G. C. Montanari, "The role of trapped space charges in the electrical aging of insulating materials," IEEE Trans. Dielectr. Electr. Insul., Vol. 4, No. 5, pp. 496-506, 1997.

[3] J. -P. Crine, "Aging and polarization phenomena in PE under high electric fields," IEEE Trans. Dielectr. Electr. Insul., Vol. 9, No. 5, pp. 697-703, 2002.

[4] J. P. Jones, J. P. Llewellyn and T.J. Lewis, "The contribution of field-induced morphological change to the electrical aging and breakdown of polyethylene," IEEE Trans. Dielectr. Electr. Insul., Vol. 12, No. 5, pp. 951-966, 2005.

[5] X. She, A. Q. Huang and R. Burgos, "Review of solid-state transformer technologies and their application in power distribution systems," IEEE J. Emerg. Sel. Topics Power Electron., Vol. 1, No. 3, pp. 186-198, 2013.

[6] H. Fan and H. Li, "High-frequency transformer isolated bidirectional DC-DC converter modules with high efficiency over wide load range for $20 \mathrm{kVA}$ solid-state transformer," IEEE Trans. Power Electron., Vol. 26, No. 12, pp. 3599-3608, 2011.

[7] S. Falcones, R. Ayyanar and X. Mao, "A DC-DC multiportconverter-based solid-state transformer integrating distributed generation and storage," IEEE Trans. Power Electron., Vol. 28, No. 5, pp. 2192-2203, 2012.

[8] T. Zhao, G. Wang, S. Bhattacharya and A. Q. Huang, "Voltage and power balance control for a cascaded H-bridge converter-based solid-state transformer," IEEE Trans. Power Electron., Vol. 28, No. 4, pp. 1523-1532, 2013.

[9] X. She, X. Yu, F. Wang and A. Q. Huang, "Design and demonstration of a $3.6-\mathrm{kV}-120-\mathrm{V} / 10-\mathrm{kVA}$ solid-state transformer for smart grid application," IEEE Trans. Power Electron., Vol. 29, No. 8, pp. 3982-3996, 2013.
[10] X. She, A. Q. Huang and R. Burgos, "Review of solid-state transformer technologies and their application in power distribution systems," IEEE J. Emerg. Sel. Topics Power Electron., Vol. 1, No. 3, pp. 186-198, 2013.

[11] T. Reghu, V. Mandloi and P. Shrivastava, "Compact high voltage, high peak power, high frequency transformer for converter type modulator applications," Rew. Sci. Instrum., Vol. 87, No. 4, 045109, 2016.

[12] M. Locatelli, R. Khazaka, S. Diaham, C. Pham, M. Bechara, S. Dinculescu and P. Bidan, "Evaluation of encapsulation materials for high-temperature power device packaging," IEEE Trans. Power Electron., Vol. 29, No. 5, pp. 2281-2288, 2013.

[13] L. Heinemann, "An actively cooled high power, high frequency transformer with high insulation capability," IEEE Appl. Power Electron. Conf. and Expo., (APEC), pp. 352-357, 2002

[14] I. Villar, U. Viscarret, I. Etxeberria-Otadui and A. Rufer, "Global loss evaluation methods for nonsinusoidally fed medium-frequency power transformers," IEEE Trans. Ind. Electron., Vol. 56, No. 10, pp. 4132-4140, 2009.

[15] I. A Gowaid, P. A Grain, A. Shehab, D. Holliday and B. Williams, "Analysis and Design of a Modular Multilevel Converter with Trapezoidal Modulation for medium and High Voltage DC-DC Transformers," IEEE Trans. Power Electron., Vol. 30, No.10, pp. $5439-5457,2015$.

[16] T. W. Dakin, "Electrical insulation deterioration treated as a chemical rate phenomenon," AIEE Trans., Vol. 67, No. 1, pp. 113$122,1948$.

[17] L. A. Dissado and A. Thabet, "Simulation of electrical ageing in insulating polymers using a quantitative physical mode," J. Phys. D: Appl. Phys., Vol. 41, No. 8, 085412. 2008

[18] L. A. Dissado, A. Thabet and S. J. Dodd, "Simulation of DC electrical ageing in insulating polymer films," IEEE Trans. Dielectr. Electr. Insul., Vol. 17, No. 3, pp. 890-897, 2010.

[19] L.A. Dissado, G. Mazzanti and G.C. Montanari, "Elemental strain and trapped space charge in thermoelectrical aging of insulating materials. Part 1: elemental strain under thermo-electricalmechanical stress", IEEE Trans. Dielectr. Electr. Insul., Vol. 8, No. 6, pp. 959-965, 2001.

[20] L.A. Dissado, G. Mazzanti and G.C. Montanari, "Elemental strain and trapped space charge in thermoelectrical aging of insulating materials. Life modeling", IEEE Trans. Dielectr. Electr. Insul., Vol. 8, No. 6, pp. 966-971, 2001

[21] L. A. Dissado and J. C. Fothergill, Electrical Degradation and Breakdown in Polymers. Peregrinus Press: London, 1992.

[22] D. M. Taylor and T. J. Lewis, "Electrical conduction in polyethylene terephthalate and polyethylene films," J. Phys. D: Appl. Phys., Vol. 4, Vo. 9, pp. 1346-1357, 1971

[23] R. Kubo, "Statistical-mechanical theory of irreversible processes. I. general theory and simple applications to magnetic and conduction problems," J. Phys. Soc. Japan., Vol. 12, No. 6, pp. 570-586, 1957

[24] L. A. Dissado, Handbook of Electronic and Photonic Materials, S. Kasap and P. Capper ed., Springer Press: New York, pp. 187-212, 2006.

[25] E. Neagu, P. Pissis, L. Apekis and J. L. Gomez Ribelles, "Dielectric relaxation spectroscopy of polyethylene terephthalate (PET) films," J. Phys. D: Appl. Phys., Vol. 30, No. 11, pp. 1551-60, 1997.

[26] J. C. Coburn and R. H. Boyd, "Dielectric relaxation in Poly(ethylene terephthalate)," Macromolecules, Vol. 19, No. 8, 2238-2245, 1986

[27] C. Alvarez, I. Sics, A. Nogales, Z. Denchev, S. S. Funari and T. A. Ezquerra, "Structure-dynamics relationship in crystallizing Poly(ethylene terephthalate) as revealed by time-resolved X-ray and dielectric methods," Polymer, Vol. 45, No. 11, pp. 3953-3959, 2004.

[28] G. Mazzanti, G. C. Montanari and L. A. Dissado, "Electrical aging and life models: the role of space charge," IEEE Trans. Dielectr. Electr. Insul., Vol. 12, No. 5, pp. 876-890, 2005

[29] L. A. Dissado, S. J. Urban and P. A. Norman, "Breakdown statistics of the space-charge ageing model for polymeric insulation," IEEE Conf. Electr. Insul. Dielectr. Phenomena (CEIDP), pp. 129-132, 1996. 
[30] L. A. Dissado, "Predicting electrical breakdown in polymeric insulators. From deterministic mechanisms to failure statistics," IEEE Trans. Dielectr. Electr. Insul., Vol. 9, No. 5, pp. 860-875, 2002.

[31] Lange's Handbook of Chemistry, J. G. Speight ed., McGraw-Hill Press: New York, 2005.

[32] L. Markey and G. C. Stevens, "Microstructural characterization of XLPE electrical insulation in power cables: determination of void size distributions using TEM,” J. Phys. D: Appl. Phys., Vol. 36, No. 20, pp. 2569-2583, 2003.

[33] A. Tzimas, S. Rowland, L. A. Dissado, M. Fu and U. H. Nilsson, "Effect of long-time electrical and thermal stresses upon the endurance capability of cable insulation material," IEEE Trans. Dielectr. Electr. Insul., Vol. 16, No. 5, pp. 1436-1443, 2009.

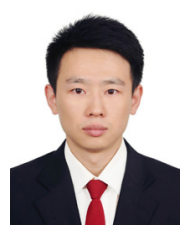

Zhou Zuo received the B.Eng. degree in electrical engineering from Chongqing University, Chongqing, China, in 2011, where he is pursuing the Ph.D. degree. His research interest is the ageing of insulating polymer under high frequency and high voltage.

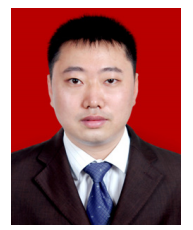

Chenguo Yao (M'08) received the B.S., M.S., and Ph.D. degrees in electrical engineering from Chongqing University, Chongqing, China, in 1997, 2000, and 2003, respectively. He became a Professor with the School of Electrical Engineering, Chongqing University, in 2007. His research interests are pulse power technology and its applications, ageing of insulation.

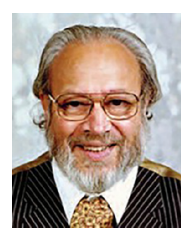

Leonard A Dissado (F'06) graduated from University College, London, with a Ph.D. in theoretical chemistry in 1966 and was awarded a D.Sc. in 1990. He published one book, together with John Fothergill, in this area in 1992. He has given numerous invited lectures, including the E.O. Forster (ICSD 2001) and the Whitehead memorial lecture (CEIDP 2002). He received the degree Doctuer Honoris Causa from the Universite Paul Sabatier, Toulouse in 2007, and a Honorary Professorship of Xian Jiaotong University, China, in 2008 .

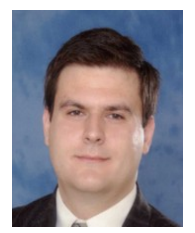

Nikola M. Chalashkanov (M'07) obtained a Masters degree from the Technical University of Sofia in 2005 and a $\mathrm{PhD}$ degree at the University of Leicester, UK in 2012. He is currently a Teaching Fellow in the Electrical Power and Power Electronics Research Group in the Department of Engineering, University of Leicester. His research interests include partial discharge and electrical treeing phenomena, dielectric properties and charge transport in polymers, chaos theory, statistical analysis and data mining. He is a member of IOP.

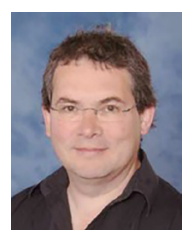

S. J. Dodd (M'11) obtained a Ph.D. degree in physics in 1992, from London Guildhall University, UK. Since 2007 he has been at University of Leicester in the Electrical Power and Power Electronics Research Group in the Department of Engineering in 2007. His research interests lie in the areas of light scattering techniques for the characterization of polymer morphology, electrical treeing breakdown process in polymeric materials and composite insulation materials, electroluminescence and its relationship with electrical and thermal ageing of polymers. 